\title{
Magnetic resonance imaging evaluation of characteristics of vascular invasion in intermediate and advanced hepatic alveolar echinococcosis
}

\author{
XIAOFEI YANG ${ }^{1 *}$, YINGLI KANG $^{1 *}$, YIJIE QIAO $^{2}$, WEIXIA LI $^{1}$, \\ JIAYUAN CAO ${ }^{1}$, HAILONG $\mathrm{LI}^{1}$ and HAIHUA BAO ${ }^{1}$ \\ ${ }^{1}$ Imaging Center of Affiliated Hospital of Qinghai University; ${ }^{2}$ Department of Radiation Oncology, \\ Medical College of Qinghai University, Xining, Qinghai 810001, P.R. China
}

Received April 25, 2018; Accepted November 14, 2018

DOI: $10.3892 /$ etm.2019.7460

\begin{abstract}
The objective of the present study was to provide a basis for the personalized treatment of intermediate and advanced hepatic alveolar echinococcosis (HAE) by elucidating the characteristics of vascular invasion and lesion growth. A total of 160 patients with intermediate and advanced HAE who were subjected to plain as well as contrast-enhanced 3.0-T magnetic resonance imaging prior to surgery were analyzed. Pathological and intra-operative observations of the subjects were also considered. The size and location of HAE lesions, vascular invasion characteristics and growth patterns were assessed. A total of 78 patients $(48.75 \%)$ had lesions involving the S5-8 segment/partial right liver lobe, 21 (13.13\%) had involvement in the S2-4 segment/partial left liver lobe and $61(38.13 \%)$ had lesions that transcended the left and right liver lobes. Pathological examination revealed that the vascular invasion rates of the hepatic portal veins, intrahepatic veins (left, central and right vein, and inferior vena cava) and hepatic arteries were $51.88,43.28$ and $26.87 \%$, respectively. Liver hilum invasion was observed in 128 patients $(80.00 \%)$, 71 of which $(44.38 \%)$ presented with invasion of the primary porta hepatis, $11(6.88 \%)$ with invasion of the secondary porta hepatis and 46 $(28.75 \%)$ with invasion of the primary as well as the secondary
\end{abstract}

Correspondence to: Professor Haihua Bao, Imaging Center of Affiliated Hospital of Qinghai University, 29 Tongren Road, Xining, Qinghai 810001, P.R. China

E-mail: baohelen2@sina.com

"Contributed equally

Abbreviations: HAE, hepatic alveolar echinococcosis; RHA, right hepatic artery; SMA, superior mesenteric artery; AA, abdominal aorta; PV, portal vein; RPV, right hepatic portal vein; LPV, left hepatic portal vein; MHV, middle hepatic vein; LHV, left hepatic vein; RHV, right hepatic vein; IVC, inferior vena cava

Key words: hepatic alveolar echinococcosis, magnetic resonance, porta hepatis, pathology porta hepatis. In conclusion, the growth pattern of intermediate and advanced HAE is determined by the site, blood supply and activity of the lesion. The current study demonstrated that lesions tend to invade the intrahepatic venous system and porta hepatis, and to target veins rather than arteries.

\section{Introduction}

Hepatic alveolar echinococcosis (HAE) is a malignant parasitic disease of the liver (1). HAE is prevalent in Asia, Africa, Europe and North America (2), and has become a serious global problem. In particular, a high incidence has been reported in Qinghai province in China (3). The current treatment strategy for HAE is with orally administered targeted drugs, which include albendazole tablets (4). However, the number of blood vessels is reduced in HAE, which results in poor drug delivery and treatment efficacy (5). Therefore, further research is required in order to understand the characteristics of vascular invasion and blood supply in HAE to provide a theoretical basis for development of novel drug administration routes and targets, as well as increasing the awareness of HAE.

Surgery is also an effective method for treating this disease $(6,7)$. However, radical resection may be difficult, as the parasite exhibits an invasive growth and tends to invade intrahepatic vessels in particular (8). Thus, accurate pre-operative assessment of a patient's vascular invasion status is key to successful surgery (9). Relatively few studies have focused on the vascular invasion status, and even fewer have addressed the physiological characteristics of vascular invasion. Furthermore, the current knowledge regarding the vascular invasion characteristics is currently insufficient and its potential significance in guiding clinical treatment remains unexplored.

Magnetic resonance imaging (MRI) is an important imaging technique that is able to display the structural characteristics of blood vessels and bile ducts in HAE, as well as their invasion status (10). Therefore, MRI of HAE lesions for analysis of vascular invasion characteristics and lesion growth patterns has an important value in increasing the accuracy of evaluation and the development of novel treatment methods. The objective of the present study was to provide a basis for personalized treatment of intermediate and advanced 
HAE by elucidating the characteristics of vascular invasion and lesion growth by using MRI, as well as intra-operative and pathological observations.

\section{Patients and methods}

Patients. A total of $160 \mathrm{HAE}$ patients treated at Affiliated Hospital of Qinghai University (Xining, China) between January 2014 and February 2017 were recruited for the present study. All of the subjects had been diagnosed with HAE by 3.0-T MRI with confirmation by post-operative pathology and had at least one lesion measuring $>5.0 \mathrm{~cm}$ in one dimension. The cohort included 79 males and 91 females. The ethnological composition of the cohort was 154 Tibetans and 6 Han Chinese individuals. The age of the patients ranged from 10 to 71 years, and their mean age was $36.17 \pm 12.15$ years. All patients underwent evaluation, analysis and surgery, with the analysis including 3.0-T MR scans.

Inclusion and exclusion criteria. The present study included patients who had i) a diagnosis of HAE based on clinical information and imaging/laboratory tests, ii) post-operative pathological tests confirming the diagnosis and iii) at least one lesion measuring $>5.0 \mathrm{~cm}$ in one dimension. Patient were excluded if they had i) comorbidities of other liver diseases, ii) severe liver or kidney impairment, iii) an allergy to the contrast agent (Ultravist), iv) concomitant conditions including pregnancy or aplastic anemia and/or v) a history of autologous liver transplantation.

MRI techniques. A Philips Achieva 3.0-T TX MR scanner (Achieva MRI; Philips, Eindhoven, the Netherlands) was used for the plain and enhanced scans using a 6-channel phased-array having two coils placed anterior to the body and a 3-channel phased-array having two coils placed posterior to the spine. Prior to scanning, patients were fasted for $4 \mathrm{~h}$, trained to hold their breathe during the scan and were requested to drink 11 of water over 30 min to distend their stomach.

MRI protocols with the following pulse sequences was used for the present study: Enhanced T1-weighted High Resolution Isotropic Volume Excitation [repetition time (TR)/echo time (TE), 3.9/1.87 msec; matrix size, 236x234; number of excitations (NEX), 1; contrast agent, magnevist ( $2 \mathrm{ml} / \mathrm{kg}$ of body weight with the flow rate of $0.2 \mathrm{ml}$, followed by normal saline flush; Chengdu, China)]; T2-weighted Spectral Attenuated Inversion Recovery (T2WI-SPAIR; TR/TE, 446/80 msec; matrix size, 268x140; section thickness/gap, 4/0.4 mm; NEX, 1); dual-Fast Field Echo (TR/TE, 4050/120 msec; matrix size, 288x174; section thickness/gap, 4/0.4 mm; NEX, 4); diffusion-weighted imaging (TR/TE, 1204/650 msec; matrix size, 256x205; NEX, 1); MR cholangiopancreatography (TR/TE, 4050/120 msec; matrix size, 288x174; section thickness/gap, 4/0.4 mm; NEX, 4). All MRI images were interpreted on a picture archiving and communications system (PACS) workstation (IntelliSpace PACS V7.0; Philips, The Netherlands)

Image processing and analysis. The raw data were transferred to the PACS and two attending physicians (WL and HL) were responsible for observing the various stages of vascular change and imaging status. They also evaluated the invasion status of i) the inferior vena cava; ii) the left, middle and right hepatic vein; iii) the trunk of the hepatic portal veins, as well as its branches; iv) the hepatic arteries; and v) the primary and secondary porta hepatis. On the basis of the MRI observations, vascular invasion was defined as follows: i) Incomplete low vascular wall signal in the enhanced T1WI-SPAIR sequence (Fig. 1); ii) hepatic artery stenosis, becoming tortuous and finer as it passed through the lesion; iii) truncation of the hepatic portal vein trunk and its branches (failure to appear on the scan) (Fig. 2); iv) hepatic veins and inferior vena cava compressed and exhibiting signs of flattening, narrowing and failure to appear on the scan (Fig. 3); v) partial or complete envelopment and stenosis of blood vessels in the primary and secondary porta hepatis (Figs. 4-6) combined with the appearance of filling defects in certain blood vessels; and vi) MR venography indicating lesions in the lumen that exhibit eccentric, irregular stenosis or occlusion (Fig. 7). Patients were excluded if they had blood vessel compression or displacement by the lesion, and if their vessels had a complete low vascular wall signal (Fig. 8).

Evaluation of pathological results. The surgeon combined MRI evaluation, intra-operative observations and gross pathology to determine the invasion status of the blood vessels prior to suturing, annotation and sending of specimens for pathological examination. The vascular walls of arteries and veins consist of (from the inside to the outside) the tunica intima, tunica media and tunica externa. On the basis of microscopic observations, vascular invasion is considered when the endothelial cells of the blood vessel are surrounded by lesion cells or covered by muscular walls with elastic membranes (Fig. 9) $(11,12)$.

Statistical analysis. The data were processed using SPSS software (version 19.0; IBM Corp., Armonk, NY, USA). All measurement data with a normal distribution were expressed as the mean \pm standard deviation, while those with a skewed distribution were presented as the median and interquartile range. The Kruskal-Wallis test was used for comparison between three groups, and the Mann-Whitney U test with Bonferroni correction was used for comparison between each group. Data were expressed as invasion rates and analyzed using $\chi^{2}$ partitioning methods, with a test level of $\alpha=0.05$, followed by Bonferroni correction. $\mathrm{P}<0.05$ was considered to indicate a statistically significant difference.

\section{Results}

Characteristics of lesions in patients with HAE. The boundary of the lesion is usually very clear (Fig. 10). In the 160 patients, a total of 181 lesions were larger than $5.0 \mathrm{~cm}$ in one dimension; the largest of these was $25.6 \times 14.4 \times 6.8 \mathrm{~cm}$ and the smallest was $5.3 \times 4.5 \times 3.2 \mathrm{~cm}$. A total of 71 lesions $(39.23 \%)$ had a maximum diameter of $5-10 \mathrm{~cm}, 76$ had a maximum diameter of $10-15 \mathrm{~cm}(41.99 \%)$ and 34 had a maximum diameter of $>15 \mathrm{~cm}(18.78 \%)$. A total of 78 patients $(48.75 \%)$ had lesions in the S5-8 segment/partial right liver lobe, 21 patients (13.13\%) had lesions involving the S2-4 segment/partial left liver lobe and 61 patients (38.13\%) had lesions that transcended the left and right liver lobes. 


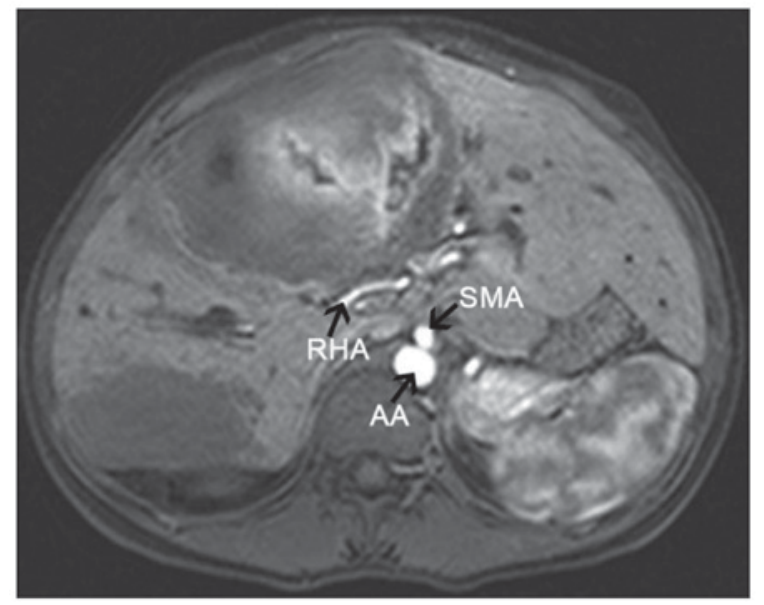

Figure 1. Transverse enhanced T1-weighted spectral attenuated inversion recovery scan indicating the disappearance of the surrounding adipose lines in the right hepatic artery. Intraoperative observation: The RHA is attached to the lesion. Pathological examination revealed slight invasion of the righ hepatic artery walls. SMA, superior mesenteric artery; AA, abdominal aorta; RHA, right hepatic artery.

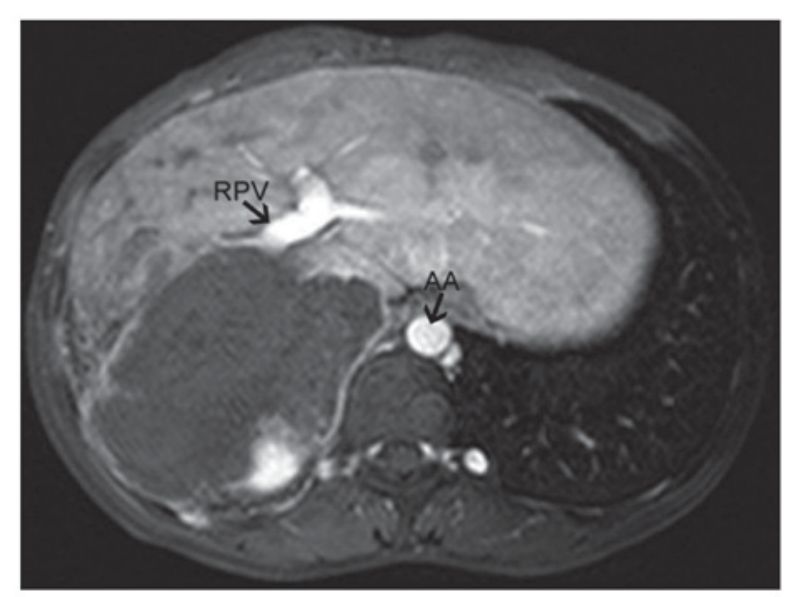

Figure 2. Transverse enhanced T1-weighted spectral attenuated inversion recovery scan indicating a blockage and absence of distal RPV on the scan. Pathological examination shows invasion of the RPV. RPV, right hepatic portal vein; AA, abdominal aorta.

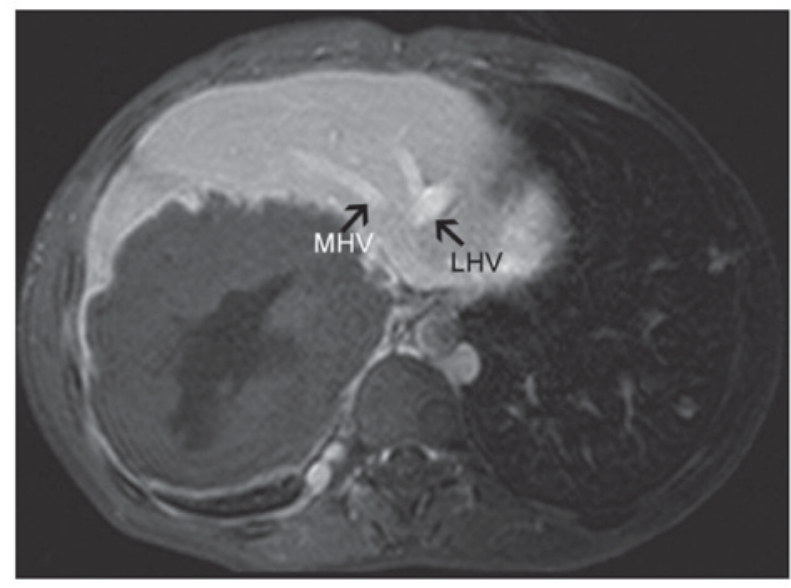

Figure 3. Transverse enhanced T1-weighted Spectral Attenuated Inversion Recovery scan with absence of the RHV and IVC. Pathological examination indicated invasion of the RHV and IVC. MHV, middle hepatic vein; LHV, left hepatic vein; RHV, right hepatic vein; IVC, inferior vena cava.

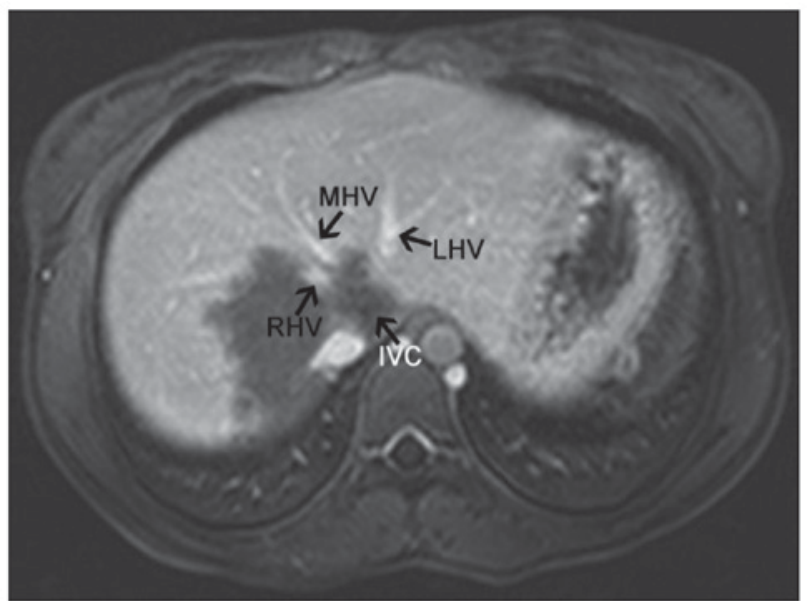

Figure 4. Transverse enhanced T1-weighted Spectral Attenuated Inversion Recovery scan indicating partial envelopment of the secondary porta hepatis. Pathological examination revealed invasion of the secondary porta hepatis, which is more significant at the exit of the RHV into the IVC. IVC, inferior vena cava; MHV, middle hepatic vein; LHV, left hepatic vein; RHV, right hepatic vein.

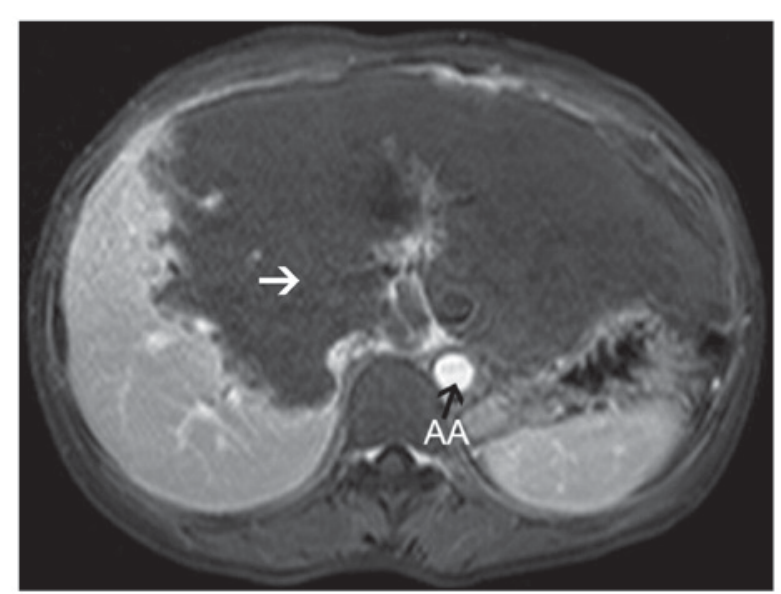

Figure 5. Transverse enhanced T1-weighted Spectral Attenuated Inversion Recovery scan demonstrating complete envelopment of the primary porta hepatis (white arrow); certain blood vessels are unclear. Pathological examination indicated severe invasion of the blood vessels and bile duct at the primary porta hepatis. AA, abdominal aorta.

Histologic analysis. Pathological examination revealed that the vascular invasion rate in the hepatic arteries, intrahepatic veins and hepatic portal veins was $26.87,43.28$ and $51.88 \%$, respectively. The cohort comprised 128 patients $(80.00 \%)$ with liver hilum invasion, 71 patients $(55.47 \%)$ featured invasion of the primary porta hepatis, 11 patients $(8.59 \%)$ had invasion of the secondary porta hepatis and 46 patients (35.94\%) exhibited invasion of the primary and secondary porta hepatis.

Data analysis results. The pathological results were regarded as the gold standard. No significant difference in age and sex was observed in either vascular invasion or hepatic hilum invasion groups (data not shown). Furthermore, no significant difference in the size of the lesions was identified between the types of hepatic vessels affected $(\mathrm{P}>0.05$; Table I). However, a significant difference was detected in the size of the lesions between the different porta hepatis invasion groups $(\mathrm{P}<0.05$; Table II). A 




Figure 6. Coronal transverse enhanced T1-weighted Spectral Attenuated Inversion Recovery scan demonstrating invasion of the primary (black arrows) and secondary (white arrows) porta hepatis. Pathological examination indicated severe invasion in the primary and secondary porta hepatis. IVC, inferior vena cava.

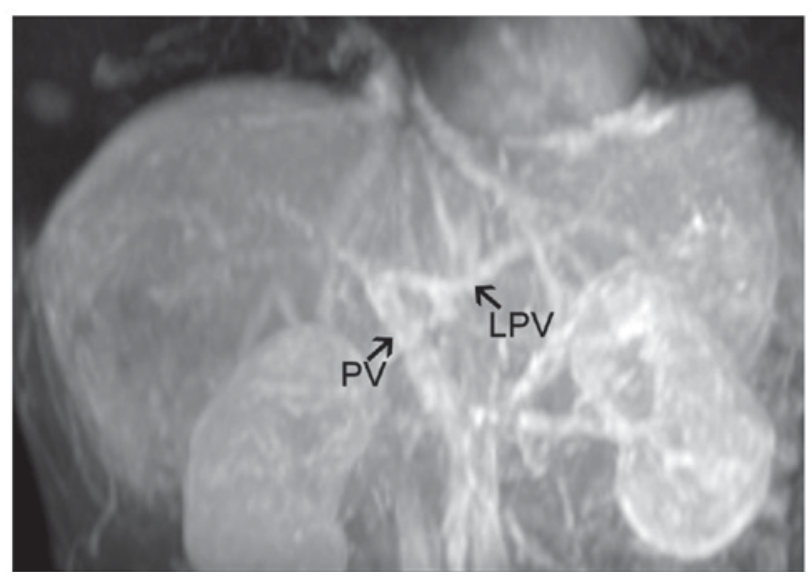

Figure 7. Coronal magnetic resonance venography indicating RPV stenosis and narrowing. Pathological examination revealed significant invasion in the RPV. R/LPV, right/left portal vein.

pairwise comparison of invasion rates between several hepatic blood vessels was performed using $\chi^{2}$-partitioning methods $\left(\alpha=0.05\right.$; Table II). A new test level $\alpha^{\prime}=0.0167$ was obtained and the difference was statistically significant $(\mathrm{P}<0.01)$. It was revealed that the invasion rate is highest in the hepatic portal veins (Table II). The maximum lesion diameter in the primary and secondary porta hepatis invasion group was significantly larger than that in the primary porta hepatis invasion group and the secondary porta hepatis invasion group, respectively $\left(\mathrm{P}<0.001\right.$; Table III). A new test level $\alpha^{\prime}=0.0167$ was obtained and the difference was statistically significant, $\mathrm{P}<0.01$. However, there was no significant difference in the maximum lesion diameter detected between the primary porta hepatis invasion group and the secondary porta hepatis invasion group $(\mathrm{P}>0.05$; Table III). A pairwise comparison of invasion rates between porta hepatis was performed using $\chi^{2}$-partitioning methods $\left(\alpha=0.05\right.$; Table IV). A new test level $\alpha^{\prime}=0.0167$ was obtained and the difference was statistically significant $(\mathrm{P}<0.01)$.

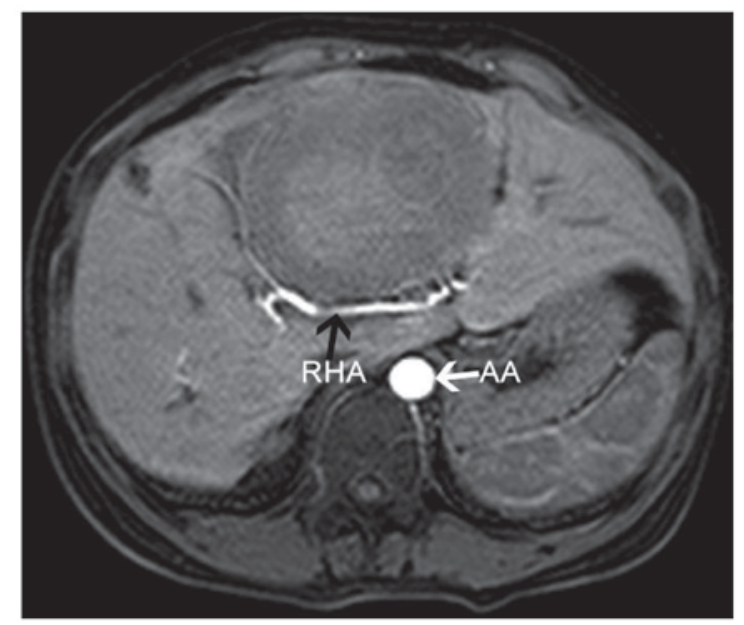

Figure 8. Transverse enhanced T1-weighted Spectral Attenuated Inversion Recovery scan displaying compression and displacement of the RHA, as well as a complete low vascular wall signal. Pathological examination indicated compression and occlusion of the RHA and absence of invasion. This is an example of a normal case that was excluded from the study. AA, abdominal aorta; RHA, right hepatic artery.

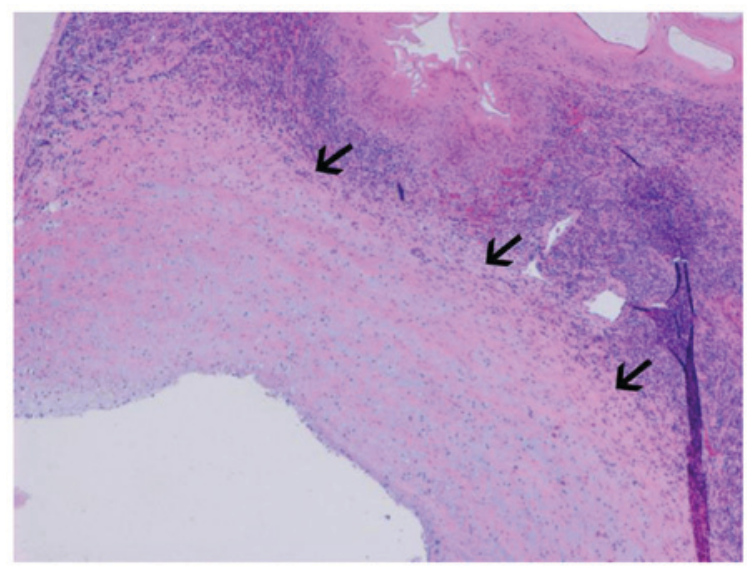

Figure 9. Magnetic resonance venography displaying eccentric stenosis of the IVC. Intraoperative observation revealed severe adhesion of the lesion and blood vessel. Pathological examination (hematoxylin and eosin; magnification, x20) demonstrated that the lesion invaded the IVC walls. Black arrows indicate the tunica externa of the blood vessel. IVC, inferior vena cava.

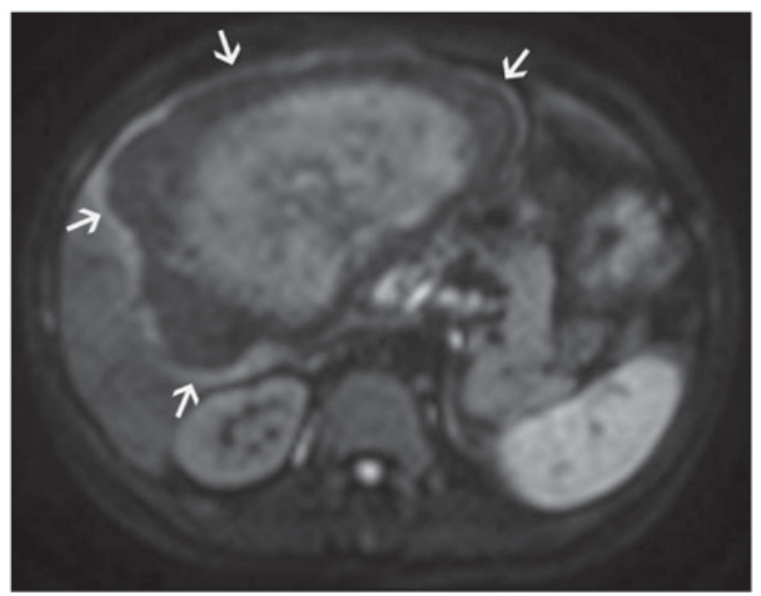

Figure 10. Transverse diffusion-weighted imaging indicating a peripheral high-signal zone surrounding the lesions; lesion boundaries (white arrows) are distinct. 
Table I. Comparison of the maximum diameter of the lesions in the intrahepatic veins, hepatic portal veins and hepatic arteries.

\begin{tabular}{lccr}
\hline Vessel type & Invaded vessels $(\mathrm{n})$ & Maximum lesion diameter $(\mathrm{cm})$ & $\chi^{2}$ \\
\hline Intrahepatic veins & 277 & $12.30(10.35-15.25)$ & $\mathrm{P}$-value \\
Hepatic portal veins & 166 & $11.65(8.98-14.60)$ & 3.84 \\
Hepatic arteries & 86 & $12.50(9.88-15.10)$ & 0.147 \\
\hline
\end{tabular}

Values are expressed as the median (interquartile range).

Table II. Pairwise comparison of invasion rates in the intrahepatic veins, hepatic portal veins and hepatic arteries.

\begin{tabular}{|c|c|c|c|c|}
\hline Vessel type & Vessels (n) & Invaded vessels, n (\%) & $\chi^{2}$ & P-value \\
\hline Intrahepatic veins & 640 & $277(43.28)$ & - & - \\
\hline Hepatic portal veins & 320 & $166(51.88)^{\mathrm{a}}$ & - & $<0.05$ \\
\hline Hepatic arteries & 320 & $86(26.87)^{\mathrm{a}, \mathrm{b}}$ & - & $<0.05$ \\
\hline Total & 1,280 & $529(41.33)$ & 43.25 & $<0.001$ \\
\hline
\end{tabular}

${ }^{\mathrm{a}} \mathrm{P}<0.05$ vs. intrahepatic veins; ${ }^{\mathrm{b}} \mathrm{P}<0.05$ vs. hepatic portal veins. Intrahepatic veins comprise the left, middle and right hepatic veins, and the inferior vena cava.

Table III. Pairwise comparison of the maximum diameter of lesions in the hepatic hilum.

\begin{tabular}{lccr}
\hline Areas affected & Cases of invasion, n $(\%)$ & Maximum lesion diameter $(\mathrm{cm})$ & $\chi^{2}$ \\
\hline Primary porta hepatis & $71(55.47)$ & $11.90(10.00-15.00)$ & \\
Secondary porta hepatis & $11(8.60)$ & $10.90(10.30-11.80)$ & 25.463 \\
Primary and secondary porta hepatis & $46(35.94)$ & $14.90(12.73-16.65)^{\mathrm{a}, \mathrm{b}}$ & $<0.001$
\end{tabular}

${ }^{\text {aP }}<0.001$ vs. primary porta hepatis; ${ }^{b} \mathrm{P}<0.001$ vs. secondary porta hepatis. Values are expressed as the median (interquartile range).

Table IV. Pairwise comparison of invasion rates in the hepatic hilum.

\begin{tabular}{lccr}
\hline Site & Total cases (n) & Cases of invasion, n (\%) & $\chi^{2}$ \\
\hline Primary porta hepatis & 160 & $71(44.38)$ & - \\
Secondary porta hepatis & 160 & $11(6.88)^{\mathrm{a}}$ & - \\
Primary and secondary porta hepatis & 160 & $46(28.75)^{\mathrm{a}, \mathrm{b}}$ & - \\
Total & 480 & $128(26.67)$ & - \\
\end{tabular}

${ }^{\mathrm{a}} \mathrm{P}<0.01$ vs. primary porta hepatis; ${ }^{\mathrm{b}} \mathrm{P}<0.01$ vs. secondary porta hepatis.

\section{Discussion}

HAE is able to invade the intrahepatic vasculature and infest tissues and organs. Late-stage HAE may result in cirrhosis, jaundice and liver failure (13), as well as metastasize to organs including the lungs and brain (14). The management of this disease is based on drugs (15), while surgery (including radical resection, lesion reduction or liver transplantation) is the major treatment method applied $(16,17)$. However, HAE lesions tend to invade blood vessels, resulting in a low success rate. Thus, simple diagnosis by imaging no longer fulfills clinical requirements, and researchers are now focusing on pre-operative vascular evaluation. In this regard, 3.0-T multi-stage enhanced MR scanning has high temporal and spatial resolution capabilities and is able to clearly display the boundaries of the lesions. This may shorten the blood T1 time and improve blood signals so that the contrast between blood vessels and surrounding tissues is enhanced and the blood vessel structure is clearly displayed. This allows clinicians to accurately evaluate the vascular invasion status and to design personalized treatment regimens (18-20). Wang et al (21) investigated vascular involvement in cystic echinococcosis. 
However, HAE reproduces by exogenous budding or infiltrative growth, and has malignant invasion characteristics (1). In this way, the disease differs from cystic echinococcosis with regard to vascular involvement. One study reported that MR angiography and MR venography are able to indicate the association between the lesion and the degree of vascular stenosis (22). In addition, according to Li et al (10), MRI is more accurate than CT with this regard. Therefore, clinicians may use MRI to examine the vascular invasion characteristics and lesion growth patterns.

In the present study, a pairwise comparison of invasion rates in the intrahepatic veins, hepatic portal veins and hepatic arteries was performed. It was revealed that the invasion rate is highest in the hepatic portal veins, followed by that in intrahepatic veins and hepatic arteries. The possible reasons for this are as follows: i) The liver has a dual blood supply, with the hepatic portal veins supplying $75 \%$ of the blood, while the hepatic arteries supply $25 \%$. Thus, the tendency of the hepatic portal veins to be invaded is increased due to the ratio of blood supply to the liver (23). ii) In the present study, lesions in the right hemiliver accounted for $49.38 \%$, while those in the left hemiliver accounted for $13.13 \%$ of all total cases. In the S-2 and S-3 segments of the left hemiliver and the S-7 and S-8 segments of the right hemiliver, the blood flow in the hepatic vein and inferior vena cava were more abundant. However, in the S-5 and S-6 segments of the right hemiliver and the S4b segment of the left hemiliver, the left and right branches of the hepatic portal vein and its tributaries contained more blood vessels. Therefore, the hepatic portal vein and its tributaries tend to be invaded more frequently. iii) Arteries are thicker than veins and have more smooth muscle and elastic fibers within their vascular walls, with smaller lumens compared with veins. Veins have thinner walls with large lumens, and are less elastic compared with arterial vascular walls. Therefore, veins are more likely to be invaded than arteries (24). iv) HAE larvae enter the liver through the mesenteric veins and hepatic portal veins. They then invade the liver tissues and vasculature, causing vesicles and cyst fluid to enter the blood vessels and metastasize to the lung, brain and other organs via hepatic venous drainage into the inferior vena cava. The transmission and dissemination route require passage through the portal venous system and are intimately associated with the tendency towards venous invasion (25). v) Baheti et al (26) reported that the invasion rate of liver cancer was greater in the hepatic portal veins than in the intrahepatic veins, and greater in the intrahepatic veins than in the hepatic arteries. They also suggested that liver cancer is mainly supplied by blood from the hepatic arteries, with the hepatic portal veins supplying only a small percentage of the blood. Conversely, Fan et al (27) and Ren and Xiao (28) indicated that the major blood supply in HAE is from the hepatic arteries, while the hepatic portal veins only provide a minor amount of blood. The vascular invasion and blood supply observed in patients with HAE appear to be similar to that of patients with liver cancer, and the vascular invasion characteristics also exhibit certain similarities.

The present study also investigated invasion in the hepatic hilum. The ratio of invasion in this type of tissue was ranked as follows: Primary porta hepatis > primary and secondary porta hepatis $>$ secondary porta hepatis. The possible reasons for this are as follows: i) The left, middle and right hepatic vein drain into the inferior vena cava through the secondary porta hepatis, while the hepatic arteries, hepatic portal veins and hepatic ducts enter the liver through the primary porta hepatis. The bifurcation of the hepatic arteries is furthest away from the hepatic hilum, while the hepatic portal vein bifurcation is slightly further from the secondary porta hepatis than the hepatic duct bifurcation. When an echinococcosis larva enters the liver through the hepatic portal vein, it is blocked by liver sinusoids and grows near the primary porta hepatis. As HAE easily invades the hepatic portal veins, a localized lesion is most likely to invade the primary porta hepatis, while the secondary porta hepatis is only invaded if the lesion grows. ii) In the present study, most lesions were situated in the right hemiliver, with the S-4 and S-5 segments being the most common sites. Lesions in this location are anatomically nearer to the primary porta hepatis and the extent of invasion is greater; iii) HAE lesions receive blood from the hepatic arteries, as well as from the hepatic portal veins. The primary porta hepatis receives the most blood from the hepatic portal veins and hepatic arteries. At the secondary porta hepatis, the hepatic veins exit the liver and contain fewer nutrients; iv) Follow-up examinations indicated that the lesion first invades the primary porta hepatis prior to growing upwards through the inferior vena cava and finally invading the secondary porta hepatis. Only in a few cases, the secondary porta hepatis was involved. Thus, the barrier effects of the liver sinusoids resulted in fewer cases where echinococcosis larvae first resided in the S-7 and S-8 segments. Conversely, the intensity of lesion activity was associated with lesion growth, which was in turn supported by portosystemic anastomosis in the hepatic portal veins and intrahepatic veins (data not shown).

The current study demonstrated that HAE has vein-targeting characteristics. Therefore, on the basis of the initial diagnosis, depending on whether the lesion is situated close to veins, clinicians can usually predict growth trends and possible factors that may affect surgical resection. These predictions may be considered to guide the selection of treatment options, including the earlier use of drugs to suppress lesion growth or direct resection. Furthermore, in patients who are treated with oral drugs, the route of administration may be changed to intravenous injection, nanoparticle implantation or targeted delivery to the hepatic portal veins, so that the drugs act rapidly on the lesions. This approach may eliminate the disadvantages associated with oral drugs, reducing the drug dosage and systemic toxicity, and increasing the therapeutic efficacy.

In case of flattened and narrowed hepatic portal veins, hepatic veins and inferior vena cava appearing on MRI examination and during surgery, the surgeon would usually perform a hemihepatectomy to dissect blood vessels from the lesions. If the hepatic artery appears narrowed and the contrast agent development of the blood vessel is partially interrupted, the possibility of invasion is greater. The secondary and tertiary blood vessels of corresponding liver segments may be resected during a hemiliver resection, reducing the intra-operative risk. Extensive involvement is indicated if the lesion has invaded the primary porta hepatis, and irregular stenosis and signs of blockade appear in the MRI of the hepatic portal vein. In such cases, the lesion is difficult to dissect during surgery. In such 
cases, extended hemihepatectomy is commonly peformed, whereby the liver segment is resected along with the walls of certain blood vessels, and the vessels are then repaired using patches or direct suturing. In case of invasion of the primary porta hepatis as well as the secondary porta hepatis, wrapping of the inferior vena cava and hepatic vein root is usually performed. In such cases, it is difficult to completely excise the lesion during surgery, and surgeons usually opt for palliative lesion reduction surgery, wherein certain parts of the lesion are left behind.

In conclusion, the growth pattern of intermediate and advanced HAE is determined by the location, blood supply and activity of the lesion. Considering its vein-targeting feature, lesions tend to invade the intrahepatic venous system and porta hepatis.

\section{Acknowledgements}

The authors wish to thank Dr Xingjian Guo (Department of Pathology, Qinghai University Affiliated Hospital, Xining, China) for helping with the histopathological analysis of the vascular invasion.

\section{Funding}

The current study was supported by a grant from the Qinghai Provincial Science and Technology Planning Project of Qinghai University Affiliated Hospital (grant no. 2017-SF-158).

\section{Availability of data and materials}

The datasets used and/or analyzed during the present study are available from the corresponding author on reasonable request.

\section{Authors' contributions}

XY and YK analyzed and interpreted all patient data regarding the status of HAE. HB designed the project and also reviewed the manuscript. HL and WL examined the degree of vascular invasion and also reviewed the manuscript. JC performed the histological examinations on hepatic tissue samples. YQ acquired imaging in patients. YQ and XY prepared the manuscript. All authors read and approved the final manuscript.

\section{Ethics approval and consent to participate}

The present study was approved by the Ethics Committee of the Affiliated Hospital of Qinghai University (approval no. P-SL-2017053). Written informed consent was obtained from the individual participants or their guardians, and all procedures conformed to the ethical standards of the China Human Experimentation Committee.

\section{Patient consent for publication}

Not applicable.

\section{Competing interests}

The authors declare that they have no competing interests.

\section{References}

1. Carmena D and Cardona GA: Echinococcosis in wild carnivorous species: Epidemiology, genotypic diversity, and implications for veterinary public health. Vet Parasitol 202: 69-94, 2014.

2. Farrokh D, Zandi B, Pezeshki Rad M and Tavakoli M: Hepatic alveolar echinococcosis. Arch Iran Med 18: 199-202, 2015.

3. Ren L, Zhang LQ, Zhou FS, Fan HN, Deng Y, Wang HJ, Ma J, Wang $Z$ and Luo SDW: Epidemiological investigation on hepatic hydatid disease in Banma county of Guoluo tibetan autonomous prefecture of Qinghai province. Chin J Dis Control Prev 20: 1032-1035, 2016 (In Chinese).

4. Mihmanli M, Idiz UO, Kaya C, Demir U, Bostanci O, Omeroglu S and Bozkurt E: Current status of diagnosis and treatment of hepatic echinococcosis. World J Hepatol 28: 1169-1181, 2016.

5. Jiang W, Wang J, Xiao H, Li TT, Liu H and Liu W: Evaluation of blood supply distribution of hepatic echinococcosis by dual-source CT energy imaging: correlation between iodine concentration and histopathology. J Xinjiang Med Univ, 38: 1207-1212, 2015 (In Chinese).

6. Fang D, Chen ZY, Zeng Y, Li B, Wen TF, Wang WT, Wu H, Xu MQ, Yang JY, Wei YG, et al: Surgical treatment of hepatic alveolar echinococcosis. Chin J Bases Clin in General Surg 23: 521-525, 2016 (In Chinese).

7. Chen KF, Tang YY, Wang R, Fang D, Chen JH, Zeng Y, Li B, Wen TF, Wang WT, Wu H, et al: The choose of different surgical therapies of hepatic alveolar echinococcosis: A single-center retrospective case-control study. Medicine (Baltimore) 97: e0033, 2018.

8. Zhang SJ, Zhang L, Zhao JQ, Zhang YG, Wu XW, Peng XY Cao YW, Yang HQ, Lv HL, Sun H and Chen XP: Inhibition of invasive growth and metastasis of hepatic alveolar echinococcosis by anti-osteopontin antibody. Chin J Parasitol Parasit Dis 29: 410-414, 2011 (In Chinese).

9. He YB, Bai L, Aji T, Jiang Y, Zhao JM, Zhang JH, Shao YM, Liu WY and Wen H: Application of 3D reconstruction for surgical treatment of hepatic alveolar echinococcosis. World J Gastroenterol 35: 10200-10207, 2015.

10. Li HL, Hou LC, Ren L, Fan HN, Bao HH, Wen SB and Li WX: Significance of magnetic resonance imaging in preoperative evaluation for patients with hepatic alveolar echinococcosis. Chin J Bases Clin in General Surg 23: 535-538, 2016 (In Chinese).

11. Sanaat Z, Khalili R, Almasi S, Aliparasti MR, Tavangar SM, Movasaghpoor A, Kazemi F and Davani A: Does chemotherapy change expression of VEGF A\&C and MVD in acute myeloid leukemia? Int J Hematol Oncol Stem Cell Res 8: 24-29, 2014.

12. Ramnefjell M, Aamelfot C, Helgeland L and Akslen LA: Vascular invasion is an adverse prognostic factor in resected non-small-cell lung cancer. APMIS 125: 197-206, 2017.

13. Geramizadeh B, Attaran Y, Malek-Hosseini SA, Kaviani MJ and Hossieni-Asl K: Photoclinic. Alveolar hydatid cyst of the liver. Arch Iran Med 14: 211-212, 2011.

14. Piarroux M, Piarroux R, Giorgi R, Knapp J, Bardonnet K, Sudre B, Watelet J, Dumortier J, Gérard A, Beytout J, et al: Clinical features and evolution of alveolar echinococcosis in France from 1982 to 2007: Results of a survey in 387 patients. J Hepatol 55: 1025-1033, 2011.

15. Vuitton DA and Bresson-Hadni S: Alveolar echinococcosis: Evaluation of therapeutic strategies. J Expert Opin Orphan Drugs 2: 67-86, 2014.

16. Nunnari G, Pinzone MR, Gruttadauria S, Celesia BM, Madeddu G, Malaguarnera G, Pavone P, Cappellani A and Cacopardo B: Hepatic echinococcosis: Clinical and therapeutic aspects. World J Gastroenterol 18: 1448-1458, 2012.

17. Wen H, Dong JH, Zhang JH, Zhao JM, Shao YM, Liang YR and Ji XW: Ex-vivo liver resection combined liver autotransplantation for the treatment of hepatic alveolar echinococcosis. Chin J Dig Surg 10: 148-149, 2011.

18. Qian NS, Liao YH, Cai SW, Rautd V and Dong JH: Comprehensive application of modern technologies in precise liver resection. Hepatobiliary Pancreat Dis Int 12: 244-250, 2013.

19. Thian YL, Riddell AM and Koh DM: Liver-specific agents for contrast-enhanced MRI: Role in oncological imaging. Cancer Imaging 13: 567-579, 2013.

20. Sun YQ, Zhang YH, Yang M, Wang JJ and Zhang QX: Application of CT and MRI in Ex-vivo autologous liver transplantation of advanced hepatic alveolar echinococcosis. Chin J Med Imaging 23: 610-613, 2015 (In Chinese). 
21. Wang J, Huang Y, Zhao YP, Liu WY, Wang J and Liu XL: Preoperative multi-slice spiral CT evaluation of involvement of vessels and biliary ducts in hepatic cystic echinococcosis. Chin J Radiol 44: 397-400, 2010.

22. Wu JW, Qu XL, Gao H, Lv MG, Lu L and Wang Xuan: Application of LAVA CE-MRA in hepatectomy for huge hepatic tumor. Chin J Prac Surg 33: 960-963, 2013.

23. Goceri E: Automatic labeling of portal and hepatic veins from MR images prior to liver transplantation. Int J Comput Assist Radiol Surg 11: 2153-2161, 2016.

24. Li Q, Lai SL, Zhang W, Jin GQ, Wang C, Su DK and Xie D: Radiologic hepatic capsular invasion on msct in prediction of microvascular invasion of hepatocellular carcinoma. J Clin Radiol 6: 838-840, 2017.

25. Conraths FJ, Probst C, Possenti A, Boufana B, Saulle R, La Torre G, Busani L and Casulli A: Potential risk factors associated with human alveolar echinococcosis: Systematic review and meta-analysis. PLoS Negl Trop Dis 11: e0005801, 2017.
26. Baheti AD, Dunham GM, Ingraham CR, Moshiri M, Lall C, Park JO, Li D, Katz DS, Madoff DC and Bhargava P: Clinical implications for imaging of vascular invasion in hepatocellular carcinoma. Abdom Radiol (NY) 41: 1800-1810, 2016.

27. Fan YX, Ren WX, Bawudun D, Ji WZ, Gu JP, Xu XD and Zhang HX: Digital subtraction angiographic observation on blood supply of rat's hepatic alveolar echinococcosis. Chin J Interv Imaging Ther 9: 37-40, 2012.

28. Ren WX and Xiao XS: The blood supply of portal vein in experimentalhepatic alveolar echinococcosis. Chin J Interv Imaging Ther 4: 142-147, 2007. 